\title{
THE FINAL VALUE PROBLEM FOR SOBOLEV EQUATIONS
}

\author{
JOHN LAGNESE
}

\begin{abstract}
Let $A$ and $B$ be $m$-accretive linear operators in a complex Hilbert space $H$ with $D(A) \subset D(B)$. The method of quasi-reversibility is used to obtain a solution to the Sobolev equation $(d / d t)[(I+B) u(t)]+A u(t)$ $=0,0<t<1$, which approximates a specified final value $u(1)=f$. In general, when $D(A) \subset D(B)$, it is not possible to find a solution which achieves exactly the final value $u(1)=f$.
\end{abstract}

1. Let $A$ and $B$ be a linear $m$-accretive operators in a complex Hilbert space $H$ with $D(A) \subset D(B)$. The purpose of the present note is to show how the method of quasi-reversibility [4] can be used to treat the final value problem

$$
\begin{gathered}
L u=(d / d t)[(I+B) u(t)]+A u(t)=0, \quad 0<t<1, \\
u(1)=f .
\end{gathered}
$$

Since this problem is not well posed, in general, when $D(A) \subset D(B)$, one may consider instead the problem of approximation of the final value, that is, given $\rho>0$, find, if possible, a solution $u_{\rho}$ of $(1.1)$ such that $\left\|u_{\rho}(1)-f\right\|<\rho$. Quasireversibility is a constructive method of determining such a solution.

In this method, one approximates the operator $L$ by a nearby operator $L_{\rho}$ such that the final value problem for $L_{\rho}$ is well posed (although the initial value problem may be ill posed; hence the term quasi-reversibility). The value $v(0)$ of the solution of $L_{\rho} v=0, v(1)=f$, is then used as an initial value in solving (1.1).

Of course, various approximating operators $L_{\rho}$ may be used. Here we approximate (1.1) by

$$
L_{\rho} v=(d / d t)[(I+B+\varepsilon A) v(t)]+A v(t)=0, \quad \varepsilon=\varepsilon(\rho) .
$$

For this choice of $L_{\rho}$ both the initial and final value problems are well posed. Furthermore, this type of approximation is stable in a sense to be made precise.

Our choice of (1.3) is suggested by the results of [6] where such an approximation procedure is used to treat the special case $B=0$. In fact, we shall show how the results of [6] can be used to obtain estimates in the general case as well.

An additional condition imposed on the operators $A$ and $B$ is a sector condition:

Received by the editors June 16, 1975.

AMS (MOS) subject classifications (1970). Primary 35R20, 35R25; Secondary 47A50. 


$$
|\arg (A x,(I+B) x)| \leqslant \pi / 4, \quad \forall x \in D(A) .
$$

In $\$ 3$ we shall give examples of how operators may be constructed which satisfy (1.5). When $B=0,(1.4)$ is equivalent to a hypothesis that the semigroup generated by $-A$ has an analytic extension into the sector $|\arg z|<\pi / 4$ of the complex plane.

2. We first consider the case $B=0 . A$ is assumed to be $m$-accretive, that is, $\operatorname{Re}(A x, x) \geqslant 0$ for all $x \in D(A)$ and $\operatorname{Rg}(I+A)=H$. By a solution of

$$
L u=d u / d t+A u=0
$$

on $[0,1]$ is meant a function $u \in C([0,1] ; H) \cap C^{\prime}((0,1) ; H)$ such that for all $t$ in $(0,1), u(t) \in D(A)$ and $(2.1)$ is satisfied.

Let $S(t), t \geqslant 0$, be the continuous semigroup of contractions on $H$ generated by $-A$ and, for each $\varepsilon>0$, let $S_{\varepsilon}(t),-\infty<t<+\infty$, be the continuous group of bounded operators on $H$ generated by the bounded, dissipative operator $-A_{\varepsilon}=\varepsilon^{-1}\left((I+\varepsilon A)^{-1}-I\right)$. Let $f \in H$ and set $v(t)=S_{\varepsilon}(t-1) f$. Then $v$ satisfies $d v / d t+A(I+\varepsilon A)^{-1} v=0$ and so is "formally" (that is, if $v \in D(A)$ and the interchange of operations is justified) a solution of the problem

$$
(d / d t)[(1+\varepsilon A) v(t)]+A v(t)=0, t<1, \quad v(1)=f .
$$

Let $u_{\varepsilon}(t)$ be the solution on $[0,1]$ of $(2.1)$ satisfying the initial condition $u_{\varepsilon}(0)=S_{\varepsilon}(-1) f$. Then $u_{\varepsilon}(t)=S(t) S_{\varepsilon}(-1) f$ and one expects $u_{\varepsilon}(1)$ to approximate $f$ in some sense. The following results are proved in [6]: Let $E_{\varepsilon}(t)$ $=S(t) S_{\varepsilon}(-t), t \geqslant 0$, and assume $A$ is $m$-sectorial with semiangle $\pi / 4$ (that is, (1.4) holds with $B=0$ ). Then

(I) $E_{\varepsilon}(t), t \geqslant 0$, is a contraction semigroup on $H$ and $E_{\varepsilon}(t) f \rightarrow f$ as $\varepsilon \rightarrow 0_{+}$ for each $f \in H$, uniformly on bounded intervals of $t$. Furthermore

$$
\begin{array}{ll}
\left\|E_{\varepsilon}(t) f-f\right\| \leqslant t\left\|A f-A_{\varepsilon} f\right\|, & f \in D(A), \\
\left\|E_{\varepsilon}(t) f-f\right\| \leqslant \varepsilon t\left\|A^{2} f\right\|, & f \in D\left(A^{2}\right) .
\end{array}
$$

(II) For each $f \in H$, (2.1) has at most one solution on [0,1] satisfying $u(1)=f$. Suppose $f=S(1) \xi$ for some (necessarily unique) $\xi \in H$. Then the final value problem has a solution $u(t)=S(t) \xi$ on $[0,1]$ and for $m=0,1, \ldots$,

$$
\begin{array}{r}
\left\|u_{\varepsilon}^{(m)}(t)-u^{(m)}(t)\right\| \leqslant(M / t)^{m}\left\|E_{\varepsilon}(1) \xi-\xi\right\|, \quad \varepsilon>0,0<t \leqslant 1, \\
\left\|u_{\varepsilon}^{(m)}(t)-u^{(m)}(t)\right\| \leqslant \varepsilon[M /(t-\delta)]^{m}\left\|A^{2} S(\delta) \xi\right\|, \\
\varepsilon>0,0<\delta<1, \delta<t \leqslant 1,
\end{array}
$$

where $M$ is a positive constant.

Now we turn to the general case $B \neq 0 . A$ and $B$ are assumed $m$-accretive with $D(A) \subset D(B)$. By a solution of $(1.1)$ on $[0,1]$ is meant a function $u:[0,1] \rightarrow D(B)$ such that $(I+B) u \in C([0,1] ; H) \cap C^{\prime}((0,1) ; H)$ and for all $t$ in $(0, T), u(t) \in D(A)$ and (1.1) is satisfied. Note that the definition requires that $u(1) \in D(B)$. 
Let $\tilde{B}$ denote the restriction of $B$ to $D(A)$ and set

$$
\tilde{A}=A(I+\tilde{B})^{-1}, \quad D(\tilde{A})=\operatorname{Rg}(I+\tilde{B}) .
$$

One verifies that a function $u$ is a solution of $(1.1)$ on $[0,1]$ if and only if $\tilde{u}=(I+B) u$ is a solution on $[0,1]$ of

$$
d \tilde{u} / d t+\tilde{A} \tilde{u}=0 .
$$

If $\operatorname{Re}(A x,(I+B) x) \geqslant 0, \forall x \in D(A)$, then $\tilde{A}$ is accretive and, moreover, $m$-accretive ([5]; c f. [3]). If the more restrictive condition (1.4) is satisfied, then $\tilde{A}$ is $m$-sectorial with semiangle $\pi / 4$.

Assume that (1.4) holds and let $\tilde{S}(t), t \geqslant 0$, be the analytic semigroup of contractions on $H$ generated by $-\tilde{A}$, and $\tilde{S}_{\varepsilon}(t),-\infty<t<t+\infty$, be the group of bounded operators on $H$ generated by $-\tilde{A}_{\varepsilon}=\varepsilon^{-1}\left((I+\varepsilon \tilde{A})^{-1}-I\right)$. If $f \in D(B)$, the function $\tilde{v}(t)=\tilde{S}_{\varepsilon}(t-1)(I+B) f$ is formally a solution on $[0,1]$ of $(2.2)$ with $A$ replaced by $\tilde{A}$, and $\tilde{v}$ satisfies $\tilde{v}(1)=(I+B) f$. Hence

$$
v(t)=(I+B)^{-1} \tilde{S}_{\varepsilon}(t-1)(I+B) f
$$

is formally a solution on $[0,1]$ of

$$
(d / d t)[(I+B+\varepsilon A) v(t)]+A v(t)=0
$$

such that $v(1)=f$. Thus we define $u_{\varepsilon}(t)$ to be the solution of $(1.1)$ on $[0,1]$ satisfying the initial condition $u_{\varepsilon}(0)=(I+B)^{-1} \tilde{S_{\varepsilon}}(-1)(I+B) f$, that is

$$
u_{\varepsilon}(t)=(I+B)^{-1} \tilde{S}(t) \tilde{S}_{\varepsilon}(-1)(I+B) f .
$$

THEOREM 2.1. Let $A$ and $B$ be m-accretive operators with $D(A) \subset D(B)$ satisfying (1.4) and suppose $f \in D(B)$. Then $u_{\varepsilon}(1) \rightarrow f$ as $\varepsilon \rightarrow 0_{+}$and the approximation procedure is stable in the sense that

$$
\left\|(I+B) u_{\varepsilon}(1)\right\| \leqslant\|(I+B) f\| \text { for all } \varepsilon>0 .
$$

Furthermore,

$$
\begin{aligned}
& \left\|u_{\varepsilon}(1)-f\right\| \leqslant \varepsilon\left\|\tilde{A}_{\varepsilon} A f\right\|, \quad f \in D(A), \\
& \left\|u_{\varepsilon}(1)-f\right\| \leqslant \varepsilon\|\tilde{A} A f\|, \quad f \in D(\tilde{A} A) .
\end{aligned}
$$

Proof. These results follow from (I) above as applied to (2.3). For example, if $f \in D(A)$ we have, since $B$ is accretive,

$$
\begin{aligned}
\left\|u_{\varepsilon}(1)-f\right\| & \leqslant\left\|(I+B)\left(u_{\varepsilon}(1)-f\right)\right\|=\left\|\tilde{S}(1) \tilde{S}_{\varepsilon}(-1)(I+B) f-(I+B) f\right\| \\
& \leqslant\left\|\tilde{A}(I+B) f-\tilde{A}_{\varepsilon}(I+B) f\right\|=\left\|A f-(I+\varepsilon \tilde{A})^{-1} A f\right\| \\
& =\varepsilon\left\|\tilde{A}_{\varepsilon} A f\right\| .
\end{aligned}
$$

Similarly, we deduce the following from (II):

THEOREM 2.2. With the hypotheses of Theorem 2.1, (1.1) has at most one 
solution on $[0,1]$ satisfying $u(1)=f$. Suppose $f=(I+B)^{-1} \tilde{S}(1)(I+B) \xi$ for some (necessarily unique) $\xi \in D(B)$. Then the final value problem has a solution $u(t)$ on $[0,1]$ and for $m=0,1, \ldots$,

$$
\begin{gathered}
\left\|u_{\varepsilon}^{(m)}(t)-u^{(m)}(t)\right\| \leqslant(M / t)^{m}\left\|\tilde{E}_{\varepsilon}(1)(I+B) \xi-(I+B) \xi\right\|, \\
\varepsilon>0,0<t \leqslant 1, \\
\left\|u_{\varepsilon}^{(m)}(t)-u^{(m)}(t)\right\| \leqslant[M /(t-\delta)]^{m}\left\|\tilde{A}^{2} \tilde{S}(\delta)(I+B) \xi\right\|, \\
\varepsilon>0,0<\delta<1, \delta<t \leqslant 1,
\end{gathered}
$$

where $\tilde{E}_{\varepsilon}(t)=\tilde{S}(t) \tilde{S_{\varepsilon}}(-t)$ and $M$ is a positive constant.

Proof. The function $\tilde{u}(t)=\tilde{S}(t)(I+B) \xi$ is a solution of $(2.3)$ on $[0,1]$ satisfying $\tilde{u}(1)=(I+B) f$; hence $u(t)=(I+B)^{-1} \tilde{u}(t)$ is a solution of $(1.1)$, (1.2) on $[0,1]$. Since $\tilde{S}(t)$ is an analytic semigroup, $\tilde{u} \in C^{\infty}((0,1] ; H)$, hence $u \in C^{\infty}((0,1] ; D(B))$ where $D(B)$ is normed with its graph norm. It follows easily that the strong $H$-derivatives, $u^{(m)}(t)$, all belong to $D(B)$ and $(I+B) u^{(m)}(t)=((I+B) u(t))^{(m)}$. Hence,

$$
\left\|u_{\varepsilon}^{(m)}(t)-u^{(m)}(t)\right\| \leqslant\left\|(I+B)\left(u_{\varepsilon}^{(m)}(t)-u^{(m)}(t)\right)\right\|=\left\|\tilde{u}_{\varepsilon}^{(m)}(t)-\tilde{u}^{(m)}(t)\right\| .
$$

The estimates therefore follow from (II) above.

3. In this section we shall show how $m$-accretive operators $A$ and $B$ satisfying the sector condition (1.4) may be constructed.

Let $C$ be a selfadjoint operator and $E(\lambda),-\infty<\lambda<+\infty$, be the corresponding resolution of the identity. A spectral measure $E$ is then determined by setting $E\left(\left(\lambda_{1}, \lambda_{2}\right]\right)=E\left(\lambda_{2}\right)-E\left(\lambda_{1}\right)$. Let $f(\lambda)$ and $g(\lambda)$ be complex valued Baire functions defined and finite $E$-almost everywhere on the real line (that is, except at most on a set of measure zero with respect to the spectral measure $E)$. One may then define operators $A$ and $B$ by setting

$$
A=\int_{-\infty}^{\infty} f(\lambda) E(d \lambda), \quad B=\int_{-\infty}^{\infty} g(\lambda) E(d \lambda)
$$

with

$$
\begin{aligned}
& D(A)=\left\{x: \int_{-\infty}^{\infty}|f(\lambda)|^{2}(E(d \lambda) x, x)<\infty\right\}, \\
& D(B)=\left\{x: \int_{-\infty}^{\infty}|g(\lambda)|^{2}(E(d \lambda) x, x)<\infty\right\} .
\end{aligned}
$$

THEOREM 3.1. Assume the following hold for all $\lambda$ in the spectrum of $C$ :

(i) $\operatorname{Ref}(\lambda) \geqslant 0, \operatorname{Reg}(\lambda) \geqslant 0$,

Then $A$ and $B$ are $m$-accretive operators satisfying the sector condition (1.4) for $x \in D(A) \cap D(B)$.

Proof. We need only apply the operational calculus of selfadjoint operators [1, Chapter XII]. Since $(E(d \lambda) x, x)$ determines a positive measure,

$$
\operatorname{Re}(A x, x)=\int_{-\infty}^{\infty} \operatorname{Re} f(\lambda)(E(d \lambda) x, x), \quad x \in D(A),
$$


is accretive if $\operatorname{Re} f(\lambda) \geqslant 0$. In addition, $A$ is closed with dense domain and

$$
\operatorname{Re}\left(A^{*} x, x\right)=\int_{-\infty}^{\infty} \operatorname{Re} \bar{f}(\lambda)(E(d \lambda) x, x), \quad x \in D\left(A^{*}\right) .
$$

Thus both $A$ and its adjoint are accretive operators, and this is sufficient to conclude that $A$ is $m$-accretive. Similary for $B$.

We also have, for $x \in D(A) \cap D(B)$,

$$
(A x,(I+B) x)=\int_{-\infty}^{\infty} f(\lambda)(1+\bar{g}(\lambda))(E(d \lambda) x, x) .
$$

Thus $|\arg (A x,(I+B) x)| \leqslant \pi / 4$ if

$$
|\arg (f(\lambda)(1+\bar{g}(\lambda)))|=|\arg f(\lambda)-\arg (1+g(\lambda))| \leqslant \pi / 4 .
$$

Of course, one also has $D(A) \subset D(B)$ if, for example, $|f(\lambda)| \geqslant|g(\lambda)|$ on the spectrum of $C$.

The operators $A$ and $B$ just constructed are known to be normal operators. Other types of $m$-accretive operators which satisfy (1.4) may be constructed from fractional powers of an $m$-accretive operator $C$ as follows: Let $0<\alpha$ $<1$ and $C^{\alpha}$ denote the indicated fractional power of $C$; if $x \in D(C)$ then

$$
C^{\alpha} x=\frac{\sin \pi \alpha}{\pi} \int_{0}^{\infty} \lambda^{1-\alpha}(C+\lambda)^{-1} C x d \lambda .
$$

The following properties of $C$ are well known (see [2], [7]): (1) $C^{\alpha}$ is $m$ sectorial with semiangle $\pi \alpha / 2$. (2) $D\left(C^{\beta}\right) \subset D\left(C^{\alpha}\right)$ if $\alpha<\beta$. (3) $C^{\alpha+\beta} x$ $=C^{\alpha}\left(C^{\beta} x\right)$ if $x \in D\left(C^{2}\right), \alpha+\beta<1$. (4) $C^{\alpha}$ commutes with every bounded operator that commutes with $C$.

Let $M$ and $N$ be positive integers and $\left\{\alpha_{n}: 1 \leqslant n \leqslant N\right\}$ and $\left\{\beta_{n}: 1 \leqslant n\right.$ $\leqslant M\}$ be real numbers such that $\alpha_{N} \geqslant \beta_{M}$ and

$$
\begin{aligned}
& 0 \leqslant \alpha_{1} \leqslant \alpha_{2} \leqslant \cdots \leqslant \alpha_{N} \leqslant 1, \\
& 0 \leqslant \beta_{1} \leqslant \beta_{2} \leqslant \cdots \leqslant \beta_{M} \leqslant 1 .
\end{aligned}
$$

Set

$$
\begin{aligned}
& A=\sum_{n=1}^{N} a_{n} C^{\alpha_{n}}, \quad D(A)=D\left(C^{\alpha_{N}}\right), \quad \forall a_{n} \geqslant 0, \\
& B=\sum_{n=1}^{M} b_{n} C^{\beta_{n}}, \quad D(B)=D\left(C^{\beta_{M}}\right), \quad \forall b_{n} \geqslant 0 .
\end{aligned}
$$

$A$ and $B$ are sectorial operators with respective semiangles $\pi \alpha_{N} / 2$ and $\pi \beta_{M} / 2, D(A) \subset D(B)$ and for $x \in D(A)$,

$$
(A x,(I+B) x)=\sum_{n, m} a_{n}\left(1+b_{m}\right)\left(C^{\alpha_{n}} x, C^{\beta_{m}} x\right) .
$$

If $x \in D\left(C^{2}\right)$, then $\left(C^{\alpha_{n}} x, C^{\beta_{m}} x\right)$ belongs to a sector $|\arg z| \leqslant(\pi / 2)\left|\alpha_{n}-\beta_{m}\right|$ as can be seen by writing, for example,

$$
\left(C^{\alpha_{n}} x, C^{\beta_{m}} x\right)=\left(C^{\alpha_{n}-\beta_{m}} C^{\beta_{m}} x, C^{\beta_{m}} x\right), \quad \alpha_{n}>\beta_{m} .
$$


Thus if $x \in D\left(C^{2}\right)$,

$$
|\arg (A x,(I+B) x)| \leqslant \pi \theta / 2
$$

where $\theta=\max _{n, m}\left|\alpha_{n}-\beta_{m}\right|$.

Suppose $x \in D(A)$ and set $x_{k}=k^{2}(C+k)^{-2} x$. Then $x_{k} \in D\left(C^{2}\right), x_{k}$ $\rightarrow x$ and for $0<\alpha \leqslant \alpha_{N}, C^{\alpha} x_{k}=k^{2}(C+k)^{-2} C^{\alpha} x \rightarrow C^{\alpha} x$ as $k \rightarrow \infty$. Thus $A x_{k} \rightarrow A x, B x_{k} \rightarrow B x$ and therefore (3.5) holds for each $x \in D(A)$. One also sees in the same way that $\left(a_{n} C^{\alpha_{n}} x, a_{m} C^{\alpha_{m}} x\right)$ lies in the right-half of the complex plane. Since $a_{n} C^{\alpha_{n}}$ and $a_{m} C^{\alpha_{m}}$ are $m$-accretive, it follows from [5] that the same is true for their sum. A simple induction argument then shows that $A$ and $B$ are $m$-accretive. We have proved

THEOREM 3.2. Suppose $\left\{\alpha_{n}\right\},\left\{\beta_{n}\right\}$ satisfy (3.1), (3.2),

$$
\alpha_{N} \geqslant \beta_{N} \text { and } \max _{n, m}\left|\alpha_{n}-\beta_{m}\right| \leqslant \frac{1}{2} \text {. }
$$

Then $A$ and $B$, defined by (3.3), (3.4) are m-accretive operators which satisfy (1.4).

\section{REFERENCES}

1. N. Dunford and J. T. Schwartz, Linear operators. II: Spectral theory. Selfadjoint operators in Hilbert space, Wiley, New York, 1963. MR 32 \#6181.

2. T. Kato, Perturbation theory for linear operators, Die Grundlehren der math. Wissenschaften, Band 132, Springer-Verlag, New York, 1966. MR 34 \#3324.

3. J. Lagnese, Existence, uniqueness and limiting behavior of solutions of a class of differential equations in Banach space, Pacific J. Math. 53 (1974), 473-485.

4. R. Lattès and J.-L. Lions, The method of quasi-reversibility, Modern Analytic and Computational Methods in Sci. and Math., no. 18, Elsevier, New York, 1969. MR 39 \# 5067.

5. R. E. Showalter, Equations with operators forming a right angle, Pacific J. Math. 45 (1973), 357-362. MR 47 \#7517.

6. - The final value problem for evolution equations, J. Math. Anal. Appl. 47 (1974), 563-572.

7. K. Yosida, Functional analysis, Die Grundlehren der math. Wissenschaften, Band 123, Academic Press, New York; Springer-Verlag, Berlin, 1965. MR 31 \#5054.

Department of Mathematics, Georgetown University, Washington, D. C. 20057 